\title{
Les Brachycentrides en Inde (Trichoptera, Integripalpia)
}

\author{
F. Schmid \\ Centre de la Recherche sur les Terres et les Ressources biologiques, Edifice K.W. Neatby, Ottawa, \\ Ontario, K1A 0C6, Canada
}

Keywords: Taxonomy, Brachycentrus, Micrasema, Trichoptera, new species, India

\begin{abstract}
The brachycentrids are represented in India by only two genera. The only species of Brachycentrus is distributed all along the Himalayan Range. Micrasema is represented by twelve species, ten of which are presently described as new. A few short taxonomic and phyletic considerations are also given.
\end{abstract}

\section{Résumé}

Les Brachycentrides ne sont représentés en Inde que par deux genres. La chaîne himalayenne n'héberge qu'une seule espèce de Brachycentrus, répartie sur toute sa longueur. Le genre Micrasema est représenté par douze espèces, dont dix sont nouvelles et dont les descriptions constituent le corps de ce travail. Quelques brèves considérations taxonomiques et phylétiques sont également offertes.

\section{Introduction}

Les Brachycentrides constituent une petite famille de Trichoptères de très large répartition holarctique et orientale. Dans une région donnée, le nombre des espèces n'est jamais très élevé. Mais il n'en est pas de même des individus. Certaines espèces éclosent en masse et donnent naissance à des nuages d'innombrables imagos.

En Inde, les Brachycentrides sont localisés dans les montagnes septentrionales de ce pays et n'y sont représentés que par deux genres, Brachycentrus Curtis, 1834, contenant une espèce déjà connue, et Micrasema McLachlan, 1876, comprenant deux es- pèces connues et dix autres décrites comme nouvelles dans ce travail.

Tous les insectes étudiés dans ce travail ont été capturés par l'auteur en Inde. Dans les descriptions spécifiques, je ne désigne que l'holotype mâle et s'il y a lieu l'allotype femelle, mais il est sous-entendu que tous les spécimens étudiés sont considérés comme paratypes. Ce matériel est déposé dans la Collection Nationale canadienne des Insectes (CNC), à Ottawa, Canada. Quelques paratypes sont également déposés dans les collections du Musée zoologique d'Amsterdam (ZMA).

\section{Genre Brachycentrus Curtis, 1834}

En Inde, ce genre n'est présent que dans l'Himalaya et n'y est représenté que par une seule espèce qui semble répartie tout le long de la chaîne: $B$. kozlovi Martynov, 1909. Cette espèce appartient au sousgenre Oligoplectrodes Martynov, 1909, ce qu'indiquent les très grands appendices préanaux entièrement fusionnés l'un à l'autre sur toute leur longeur, en une plaque massive et horizontale.

\section{Brachycentrus kozlovi Martynov, 1909}

Cette espèce a été décrite par Martynov (1909) du Tibet oriental (Kham), puis signalée de la vallée du Cachemire et du Ladakh, par Mosely (1938). Ensuite, elle a été capturée dans l'Himalaya et le 
Karakoram du Pakistan, par moi-même (1961), et dans l'Indou-Kouch oriental, par Klapperich (Schmid, 1963).

Elle m'a paru assez répandue au Pauri Garhwal, où je l'ai rencontrée du début de juin à mi-août, entre 1400 et $2700 \mathrm{~m}$ d'altitude. Elle fréquente surtout les moyens torrents très agités, mais également les ruisseaux moyens et petits et plus ou moins marécageux. On la capture en général à la lumière, mais les récoltes au filet de jour et au crépuscule ne sont pas rares. Je ne l'ai pas rencontrée au Sikkim, mais la connais d'une station du Kameng, torrent très agité, vers $2400 \mathrm{~m}$, où elle est venue voler à la lumière.

\section{Genre Micrasema McLachlan, 1876}

Ce genre est assez bien représenté en Inde septentrionale, c'est-à-dire dans l'Himalaya et les montagnes para-himalayennes de l'Assam. J'en ai capturé dix espèces, ce qui, avec M. punjaubi Mosely, 1938, du Penjab, et $M$. nepalicum Botosaneanu, 1976, du Népal, qui n'ont pas été retrouvées, porte le nombre des espèces indiennes à onze.

Les insectes sont d'aspect très uniforme et ont une coloration entièrement gris noir. La longueur de l'aile antérieure varie de 4 à $5,5 \mathrm{~mm}$ seulement. La nervulation aussi est très stable. Dans les descriptions qui suivent, je ne cite guère que les caractères génitaliens du mâle. L'appareil phallique ne présente guère de caractères intéressants. Sur les figures de ce travail, l'endothèque est représentée tantôt dévaginée, tantôt invaginée dans la phallothèque et alors invisible. Ceci est dû au hasard des préparations microscopiques et de l'état physiologique des insectes lors de leur capture.

Ajoutons que les femelles de nombreuses espèces sont pourvues d'une dense pilosité au centre de la face inférieure des ailes postérieures. La fonction de ces soies semble être de s'aglomérer à la masse ovigère que les insectes portent quelque temps avant de la déposer, peut-être afin de la rendre moins adhésive avant son abandon dans l'eau.

Les caractères écologiques sont également peu variés. Les Micrasema peuplent surtout les ruisseaux agités, de taille moyenne, entre 200 et $3000 \mathrm{~m}$ d'altitude. Elles se capturent en général au filet, mais viennent occasionnellement à la lumière. Pour éviter trop de répétitions, dans les descriptions spécifiques, je n'indique, comme caractères écologiques, que les intervalles saisonniers des captures.

Taxonomiquement, nous pouvons remarquer que $M$. apratitam, $M$. dabhram et $M$. asajjanam ont leurs appendices inférieurs bi-articulés, de grands appendices préanaux en toit et plus ou moins fusionnés l'un à l'autre et le Xe segment simple. Le bord latéral apical du IXe segment est recourbé vers l'intérieur et forme un grand angle aigu flanquant la phallocrypte. Ce sont là des caractères primitifs au niveau du genre. $M$. adhiram, $M$. avadhiritam, $M$. karunam et $M$. adhacharam montrent des appendices préanaux assez petits et largement séparés l'un de l'autre. C'est là un caractère fort inattendu. La plupart des Brachycentrides ont de grands appendices préanaux triangulaires, en position dorsale et fusionnés l'un à l'autre en une plaque plus ou moins bifide, ou réunis l'un à l'autre par des membranes. Or, chez le genre le plus primitif de la famille, Eobrachycentrus Wiggins, 1965, les appendices préanaux sont en petites verrues et largement distants l'un de l'autre. Ceci est un caractère primitif au niveau ordinal. Il est donc étonnant que l'on retrouve un caractère aussi fondamentalement primitif chez certaines espèces seulement d'un genre aussi spécialisé et homogène que Micrasema. Chez les quatre espèces citées plus haut, l'écartement des appendices préanaux est donc probablement à interpréter comme une spécialisation secondaire et un retour à l'état primitif. $M$. jihmam, $M$. kripanam et $M$. abhavyam sont isolées des précédentes. Remarquons également que $M$. avadhiritam et $M$. adhacharam ont une pointe ventrale sous le VIe sternite, alors que les autres espèces en sont dépourvues.

Comme chez bien d'autres lignées de Trichoptères, le nombre des espèces est faible dans l'Himalaya occidental et augmente progressivement lorsqu'on se déplace vers l'est. Ainsi, les Micrasema sont absentes de l'Himalaya du Pakistan, représentées au Penjab et au Garhwal par une seule espèce, alors que le Sikkim, le Kameng et les montagnes de l'Assam en hébergent chacun quatre. Les Micrasema n'ont pas franchi la trouée 
des Garo-Rajmahal et ne se sont pas dispersées dans les Ghâts occidentales.

En 1989 (: 109-110, Fig. 252), j'ai indiqué que beaucoup des lignées primitives, monticoles et sténothermes froides des Trichoptères néarctiques sont originaires de la région orientale. $\mathrm{Qu}$ 'en est-il des Micrasema? La phylogénie de ce genre reste à reconstituer et l'assignation des parentés des espèces orientales avec les faunes européenne et néarctique à déterminer. Mais, à première vue, il ne semble pas que les Micrasema orientales comptent parmi elles les espèces les plus primitives du genre. Toutefois, comme il est indiqué plus haut, quatre espèces orientales montrent de petits appendices préanaux largement écartés. Ceci pourrait être un caractère primitif, à moins qu'il ne soit à interpréter différemment.

\section{Micrasema apratitam n. sp. (Figs. 1-5)}

Matériel. - Holotype $\sigma^{\prime \prime}$ et allotype $Q$ : Inde, Assam, NEFA, Kameng Frontier Division, Chug; 17-IV-1961 (CNC 21296). Paratypes dans CNC et ZMA.

Description. - Palpes maxillaires du mâle très longs, avec leur extrémité atteignant le niveau du $6 \mathrm{e}$ article des antennes, les $2 \mathrm{e}$ et $3 \mathrm{e}$ articles étant spécialement longs, grêles et arqués vers le haut.

Nervulation: chez le mâle, cellule discoïdale des ailes antérieures 1,5 fois plus longue que large; aux ailes postérieures, $\mathrm{SR}$ tribranché et $\mathrm{M}$ simple. Chez la femelle, cellule discoïdale des ailes antérieures trois fois plus longue que large; aux ailes postérieures, SR quadri-branché et $\mathrm{M}$ double. VIe sternite du mâle sans pointe ventrale. Longueur de l'aile antérieure: 5-8 $\mathrm{mm}$.

Génitalia $O^{\prime}$ (Figs. 1-5): IXe segment assez allongé latéralement et avec ses deux angles latéraux, antérieur et postérieur, bien marqués et situés au même niveau; son bord latéral apical est replié vers l'intérieur et forme un angle droit encadrant la phallocrypte. Appendices préanaux apparaissant très obtusément hémisphériques et à très large base, vus de profil; vus de dessus, ils se montrent plus anguleux et séparés l'un de l'autre par un étroit espace empli de membranes. Xe segment apparaissant comme une simple bande régulière et inclinée vers le bas, vu de profil; vu de dessus, il se montre com- me une très large languette arrondie; il y a quelques petits tubercules basaux médians spinifères; le bord du segment est faiblement spineux; vu de face, il se montre faiblement bilobé. Appendices inférieurs bi-articulés et d'épaisseur moyenne; ils sont dilatés jusqu'à leur extrémité qui se termine en deux lobes: un inférieur arrondi et sclérotisé et un supérieur, plus petit et désclérotisé; entre eux deux, le second article est visible sous la forme d'un minuscule bouton. Phallothèque avec sa face dorsale sclérotisée sur presque la même longueur que la face inférieure, ce qui fait qu'elle paraît échancrée, vue de profil.

Remarques. - $M$. apratitam (sanscrit, triste) se reconnaît immédiatement à ses très longs palpes maxillaires, à son $\mathrm{Xe}$ segment simple et linguiforme, à ses appendices inférieurs bilobés et à l'apex de la phallothèque apparaissant échancré latéralement.

Cette espèce est commune et largement répandue en Kameng, où je l'ai capturée du début d'avril à fin octobre, au filet et à la lumière. Parfois un très grand nombre de femelles accourent à la lumière.

Micrasema dabhram n. sp. (Figs. 6-10)

Matériel. - Holotype $\sigma^{\circ}$ et allotype $\$$ : Inde, Pauri Garhwal, Gaurikund; 9/10-V-1958 (CNC 21300). Paratypes dans CNC et ZMA.

Description. - Palpes maxillaires du mâle pas très longs, avec leur extrémité atteignant le niveau du $3 \mathrm{e}$ article des antennes.

Nervulation: aux ailes antérieures, cellule discoïdale trois fois plus longue que large et avec un long parcours commun avec la Fl, et cela chez les deux sexes. Aux ailes postérieures, $\mathrm{SR}$ est tribranché et $\mathbf{M}$ simple chez le mâle. Chez la femelle, SR est également tribranché et $M$ double. VIe sternite du mâle sans pointe ventrale. Longueur de l'aile antérieure: 4-5,5 mm.

Génitalia O* (Figs. 6-10): IXe segment assez court latéralement et avec ses deux angles latéraux, antérieur et postérieur, différemment marqués et situés à des niveaux très différents l'un de l'autre; son bord latéral apical est replié vers l'intérieur et forme un long angle très aigu dont la pointe atteint 

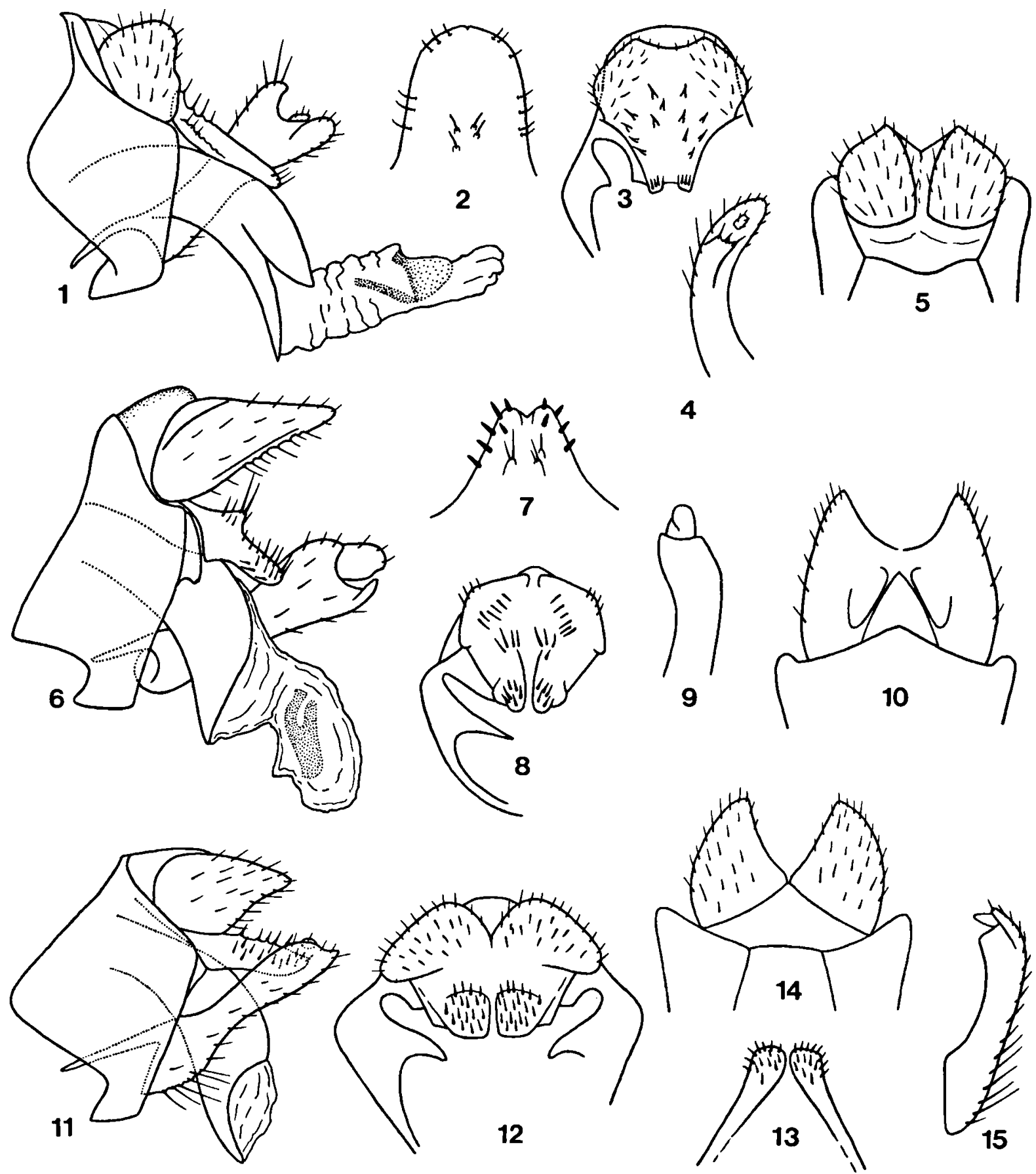

Figs. 1-5. Micrasema apratitam n. sp.: 1, génitalia de profil; 2, Xe segment de dessus; 3, Xe segment de face; 4, appendice inférieur de dessus; 5 , appendices préanaux de dessus.

Figs. 6-10. Micrasema dabhram n. sp.: 6, génitalia de profil; 7, Xe segment de dessus; 8, Xe segment de face; 9, appendice inférieur de dessus; 10, appendices préanaux de dessus.

Figs. 11-15. Micrasema asajjanam n. sp.: 11, génitalia de profil; 12, Xe segment de face; 13, Xe segment de dessus; 14, appendices préanaux de dessus; 15 , appendice inférieur de dessous. 
le bord de la phallocrypte. Appendices préanaux apparaissant en grands avant-toits triangulaires et à pointe aiguë, vus de profil; vus de dessus, ils se montrent également triangulaires et fusionnés l'un à l'autre sur leur moitié basale. Vu de profil, le Xe segment apparaît comme un gros lobe incliné vers le bas et formant deux angles médians, supérieur et inférieur; vu de dessus, il se montre comme un trapèze légèrement bilobé, pourvu de quelques grêles tubercules médians sétifères et de fortes épines latérales et apicales; vue de face, son extrémité apparaît bilobée et spinifère. Appendices inférieurs bi-articulés et d'épaisseur moyenne; leur bord apical est concave, leur angle apical inférieur en pointe grêle et leur angle apical supérieur aigu. Le second article est relativement grand et en ovale peu allongé. Phallothèque épaisse et tronquée très obliquement à son extrémité.

Remarques. - M. dabhram (sanscrit, précaire) se reconnaît à ses grands appendices préanaux en toit et partiellement fusionnés, à son Xe segment bilobé et spineux et au second article des appendices inférieurs relativement grand. Elle est assez voisine de $M$. asajjanam n. sp. par ses grands appendices préanaux en toit. Il n'est pas impossible que $M$. dabhram soit synonyme de $M$. punjaubi Mosely, 1938, qui a été très incomplètement décrite. En attendant plus de précisions sur ce sujet, je préfère risquer un synonyme plutôt que deux homonymes. M. nepalicum Botosaneanu, 1976, n'en est pas éloignée.

Cette espèce m'est connue de plusieurs localités du Pauri Garhwal, toutes situées sur le versant méridional du massif de Kedarnath, où je l'ai capturée en mai seulement. Elle semble monter en altitude plus haut que les autres espèces, jusque vers $3000 \mathrm{~m}$. Je la connais aussi par un mâle unique du Sikkim.

Micrasema asajjanam n. sp. (Figs. 11-15)

Matériel. - Holotype O $^{\prime}$ et allotype $९$ : Inde, Assam, NEFA, Kameng Frontier Division, Rahung; 25-IV-1961 (CNC 21302). Paratypes dans CNC et ZMA.

Description. - Palpes maxillaires du mâle pas très longs, avec leur extrémité atteignant le niveau du $3 \mathrm{e}$ article des antennes.
Nervulation: chez les deux sexes, aux ailes antérieures, cellule discoïdale deux fois plus longue que large et A2 perdue. Aux ailes postérieures, chez le mâle, $S R$ est tribranché et $M$ simple. Chez la femelle, SR est tribranché et $M$ double. VIe sternite du mâle sans pointe ventrale. Longueur de l'aile antérieure: $4,5-5,5 \mathrm{~mm}$.

Génitalia $O^{\prime \prime}$ (Figs. 11-15): IXe segment bien allongé latéralement et avec ses deux angles latéraux, antérieur et postérieur, bien marqués, le second étant situé un peu au-dessus du premier; son bord latéral apical est replié vers l'intérieur et forme un grand angle aigu, un peu recourbé vers le bas et dont la pointe atteint le bord de la phallocrypte. Appendices préanaux apparaissant en grands triangles irréguliers, obtus mais à pointe élancée, vus de profil; vus de dessus, ils se montrent triangulaires et à bords latéraux arqués et n'ont qu'un point en commun l'un avec l'autre; vus de face, ils apparaissent plus larges que hauts. Xe segment se montrant en lobe horizontal, pointu et spineux, vu de profil; vu de dessus, il apparaît trapézoïdal, avec ses bords latéraux sclérotisés; l'extrémité est nettement bilobée et spineuse; vus de face, ses deux lobes apicaux se montrent arrondis et spinifères. Appendices inférieurs bi-articulés et assez grêles, surtout au milieu de leur longueur; leur bord apical est sinueux et pointu; le second article est un lobe minuscule, mais bien visible. Phallothèque assez fortement échancrée à son extrémité.

Remarques. - M. asajjanam (sanscrit, vaurien) est assez voisine de $M$. dabhram n. sp., par ses grands appendices préanaux triangulaires et tectiformes et par son Xe segment clivé apicalement et fortement spineux. Elle est également voisine de $M$. baitina Mosely, 1938, par la forme du second article des appendices inférieurs, mais s'en distingue par ses appendices préanaux bien distincts l'un de l'autre.

Cette espèce ne m'est connue que du Kameng, où je l'ai capturée à un petit nombre de localités, de mi- à fin avril.

Micrasema adhiram n. sp. (Figs. 16-22)

Matériel. - Holotype O*: Sikkim, Tikjak; 7-IV-1959 (CNC 21301). Paratypes dans CNC et ZMA. 
Description. - Palpes maxillaires du mâle pas très longs, avec leur extrémité atteignant le niveau du $2 \mathrm{e}$ article des antennes.

Nervulation: cellule discoïdale des ailes antérieures deux fois plus longue que large. Aux ailes postérieures, chez le mâle, SR est tribranché et $M$ simple; chez la femelle, SR est tribranché et $M$ double. VIe sternite du mâle sans pointe ventrale. Longueur de l'aile antérieure: 4-4,5 $\mathrm{mm}$.

Génitalia $O^{\circ}$ (Figs. 16-22): IXe segment avec son angle latéral apical très peu marqué et son angle latéral antérieur largement arrondi; son bord latéral apical est à peine replié vers l'intérieur et ne forme pas d'angle proéminent encadrant la phallocrypte. Appendices préanaux petits, largement distants l'un de l'autre et non réunis par une zone membraneuse; vus de profil, ils apparaissent en petits ovales, avec un léger angle rentrant en position subapicale inférieure; vus de face, ils se montrent en triangles approximatifs et irréguliers. Xe segment petit et peu proéminent; vu latéralement, il apparaît comme un ergot recourbé vers le haut et surmonté d'un tubercule peu sclérotisé en position élevée entre les appendices préanaux; vus de face, les ergots se montrent divergents et dirigés vers le haut et les tubercules peu sclérotisés convergents l'un vers l'autre; les angles latéraux inférieurs du Xe segment apparaissent bien marqués en vue caudale. Appendices inférieurs uni-articulés et se montrant régulièrement assez épais en vue latérale; ils apparaissent se terminer en deux pointes triangulaires, l'une étant deux fois plus grande que l'autre; en vue caudale, la pointe supérieure se montre en large lobe et la pointe inférieure en ergot recourbé sous celui-là. Phallothèque recourbée à angle droit vers le bas.

Remarques. - $M$. adhiram (sanscrit, craintif) est facile à reconnaître à ses petits appendices préanaux largement écartés et à la forme générale du Xe segment et de l'apex des appendices inférieurs. C'est probablement de $M$. karunam n. sp. qu'elle se rapproche le plus par la forme du Xe segment. Quelques variations intraspécifiques sont illustrées par les figures 20-22.

Cette espèce ne m'est connue que du Sikkim, où je l'ai capturée en avril, mai, août et octobre.
Micrasema karunam n. sp. (Figs. 23-26)

Matériel. - Holotype $O^{\circ}$ et allotype $\$$ : Inde, Assam, Manipour, Chingsao; 14-VI-1960 (CNC 21297). Paratypes dans CNC et ZMA.

Description. - Palpes maxillaires du mâle pas très longs, avec leur extrémité atteignant le niveau du $2 \mathrm{e}$ article des antennes.

Nervulation: chez le mâle, cellule discoïdale des ailes antérieures 2,5 fois plus longue que large et trois fois chez la femelle. Aux ailes postérieures, SR est tribranché et $M$ simple chez le mâle; chez la femelle, SR est tribranché et chez le mâle double. VIe sternite du mâle sans pointe ventrale. Longueur de l'aile antérieure: 4-5 $\mathrm{mm}$.

Génitalia $O$ (Figs. 23-26): IXe segment très allongé latéralement et avec ses angles latéraux, antérieur et postérieur, très inégaux en obtusité et en position; son bord latéral postérieur n'est pas replié vers l'intérieur et ne forme pas d'angle proéminent encadrant la phallocrypte. La partie dorsale du IXe segment forme une courte languette ovale dirigée vers l'arrière. Appendices préanaux petits, largement distants l'un de l'autre et non réunis par une zone membraneuse; vus de profil, ils apparaissent comme de petits triangles aux angles arrondis et pourvus d'une légère échancrure préapicale inférieure; vus de face, ils se montrent légèrement bilobés. Xe segment petit, trapu et pluribranché; vu de profil, il apparaît triangulaire, terminé par un petit lobe aigu et pourvu d'un autre grand lobe basal supérieur arrondi; latéralement à ces derniers sont insérées deux petite pointes de tailles inégales; vues de dessus, ces quatre parties sont bien visibles, car elles se montrent assez distantes les unes des autres. Appendices inférieurs uni-articulés, moyennement épais et pas très longs; vus de profil, ils se montrent tronqués à l'apex et avec leur bord supérieur assez fortement sinueux; vu de face, leur bord externe se montre hémicirculaire. Phallothèque fortement arquée vers le bas, obtuse à son extrémité et tronquée droit à l'apex de sa face dorsale.

Remarques. - M. karunam (sanscrit, piteux) est surtout caractéristique par son Xe segment multilo- 

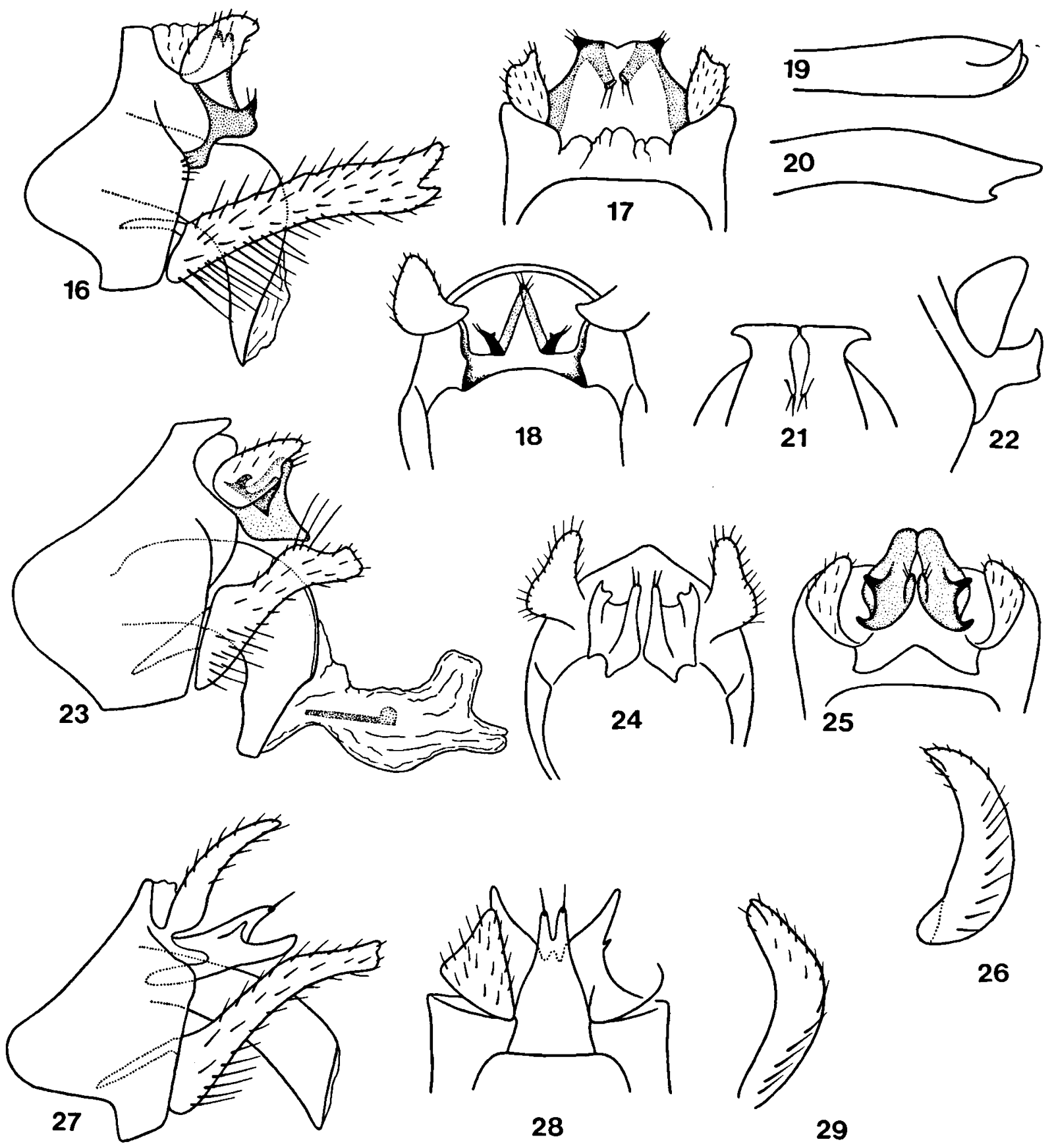

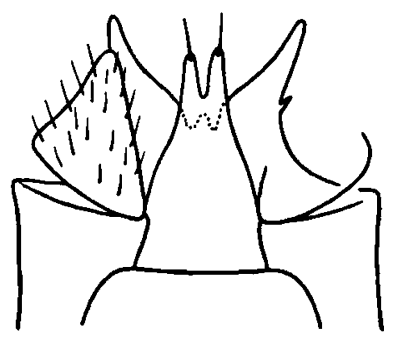

28
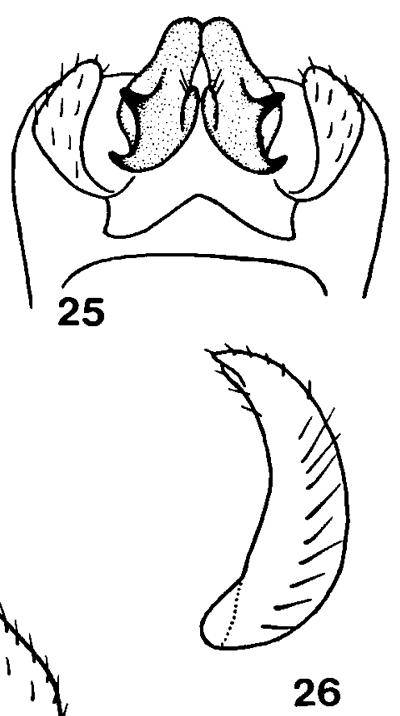

26

Figs. 16-22. Micrasema adhiram n. sp.: 16, génitalia de profil; 17, Xe segment de dessus; 18, Xe segment de face; 19, appendice inférieur de dessous; 20, appendice inférieur de profil; 21, Xe segment de dessus; 22, idem, de profil.

Figs. 23-26. Micrasema karunam n. sp.: 23, génitalia de profil; 24, Xe segment de face; 25, Xe segment de dessus; 26, appendice inférieur de dessous.

Figs. 27-29. Micrasema adhacharam n. sp.: 27, génitalia de profil; 28, Xe segment de dessus; 29, appendice inférieur de dessous. 
bulé et la grande convexité médiane préapicale supérieure des appendices inférieurs.

J'ai capturé cette espèce dans plusieurs localités de l'Etat de Manipour, en juin et juillet.

Micrasema adhacharam n. sp. (Figs. 27-29)

Matériel. - Holotype $\sigma^{\circ}$ : Inde, Assam, Manipour, Matiyang; 17-VI-1960 (CNC 21304). Paratypes dans CNC.

Description. - Palpes maxillaires du mâle pas très longs, avec leur extrémité atteignant le niveau du $2 \mathrm{e}$ article des antennes.

Nervulation: chez le mâle, cellule discoïdale environ 2,5 fois plus longue que large. Aux ailes postérieures, SR tribranché et $M$ simple. VIe sternite du mâle avec une pointe ventrale conique. Longueur de l'aile antérieure: $4 \mathrm{~mm}$.

Génitalia $\sigma^{\circ}$ (Figs. 27-29): IXe segment avec son angle latéral postérieur peu marqué et en position assez élevée et son angle latéral antérieur largement arrondi et très proéminent, ce qui donne une assez grande longueur au segment; son bord latéral apical ne forme pas d'angle saillant encadrant la phallocrypte. Appendices préanaux largement distants l'un de l'autre et non réunis par une zone membraneuse; vus de profil, ils apparaissent comme de longs croissants grêles, graduellement atténués et dressés verticalement; vus de dessus, ils se montrent assez régulièrement triangulaires. Xe segment apparaissant comme une pièce subhorizontale, vu de profil et divisé en trois paires de branches de tailles très inégales; vu de dessus, apparaît un lobe dorsal longuement triangulaire et bifide, chaque pointe portant une soie; il y a aussi deux branches latérales inférieures, largement distantes l'une de l'autre, en triangles aigus et aux bords latéraux concaves; en outre, sont visibles deux petites pointes latérales insérées sur les précédentes. Appendices inférieurs uni-articulés, longs, grêles, un peu arqués vers l'arrière et apparaissant, vus de profil, terminés en deux petits lobes peu marqués; vus de face, le lobe inférieur se montre en éperon et le lobe supérieur en ailette arrondie. Phallothèque pas très épaisse et tronquée droit à son extrémité. Femelle inconnue.
Remarques. - M. adhacharam (sanscrit, humble) n'est voisine d'aucune autre espèce indienne, mais par ses appendices préanaux en grands croissants grêles, elle rappelle $M$. hanasensis Tsuda, 1942, du Japon. Elle est caractéristique surtout par la forme du Xe segment.

Cette espèce ne m'est connue que d'une localité de Manipour et d'une autre des Lushai Hills.

Micrasema avadhiritam n. sp. (Figs. 30-33)

Matériel. - Holotype $\sigma^{\prime}$ et allotype $\bigcirc$ : Inde, Assam, United Jaintia and Khasi Hills, Syndai; 14-XII-1959 (CNC 21303). Paratypes dans CNC.

Description. - Palpes maxillaires du mâle pas très longs, avec leur extrémité atteignant le niveau du $2 \mathrm{e}$ article des antennes.

Nervulation: chez le mâle, cellule discoïdale des ailes antérieures 2,5 fois plus longue que large et 3,5 chez la femelle. Aux ailes postérieures, SR est tribranché et $\mathrm{M}$ simple chez le mâle; chez la femelle, SR est tribranché et $M$ double. VIe sternite du mâle avec une pointe ventrale conique. Longueur de l'aile antérieure: $4-5,5 \mathrm{~mm}$.

Génitalia Oo (Figs. 30-33): IXe segment assez bien allongé latéralement, avec son angle latéral postérieur bien saillant et situé haut et son angle latéral antérieur largement arrondi et en situation plus basse; son bord latéral apical ne forme pas d'angle saillant encadrant la phallocrypte, mais constitue une saillie adossée aux angles latéraux du $\mathrm{Xe}$ segment. Appendices préanaux assez grands, largement distants l'un de l'autre et non réunis par une zone membraneuse; vus latéralement, ils apparaissent comme de longs rectangles dont l'angle apical inférieur est échancré; vue de dessus et de face, cette échancrure est toujours bien visible. Xe segment en forme de V; il forme deux lobes basaux, hauts, subparallèles et assez proches l'un de l'autre et deux lobes apicaux, plus petits, plus courts, plus bas, nettement écartés l'un de l'autre et divergents. Appendices inférieurs uni-articulés, très longs et régulièrement grêles; leur extrémité est simplement recourbée vers l'intérieur et ne forme pas de lobes apicaux. Phallothèque peu arquée vers le bas et tronquée droit à son extrémité. 


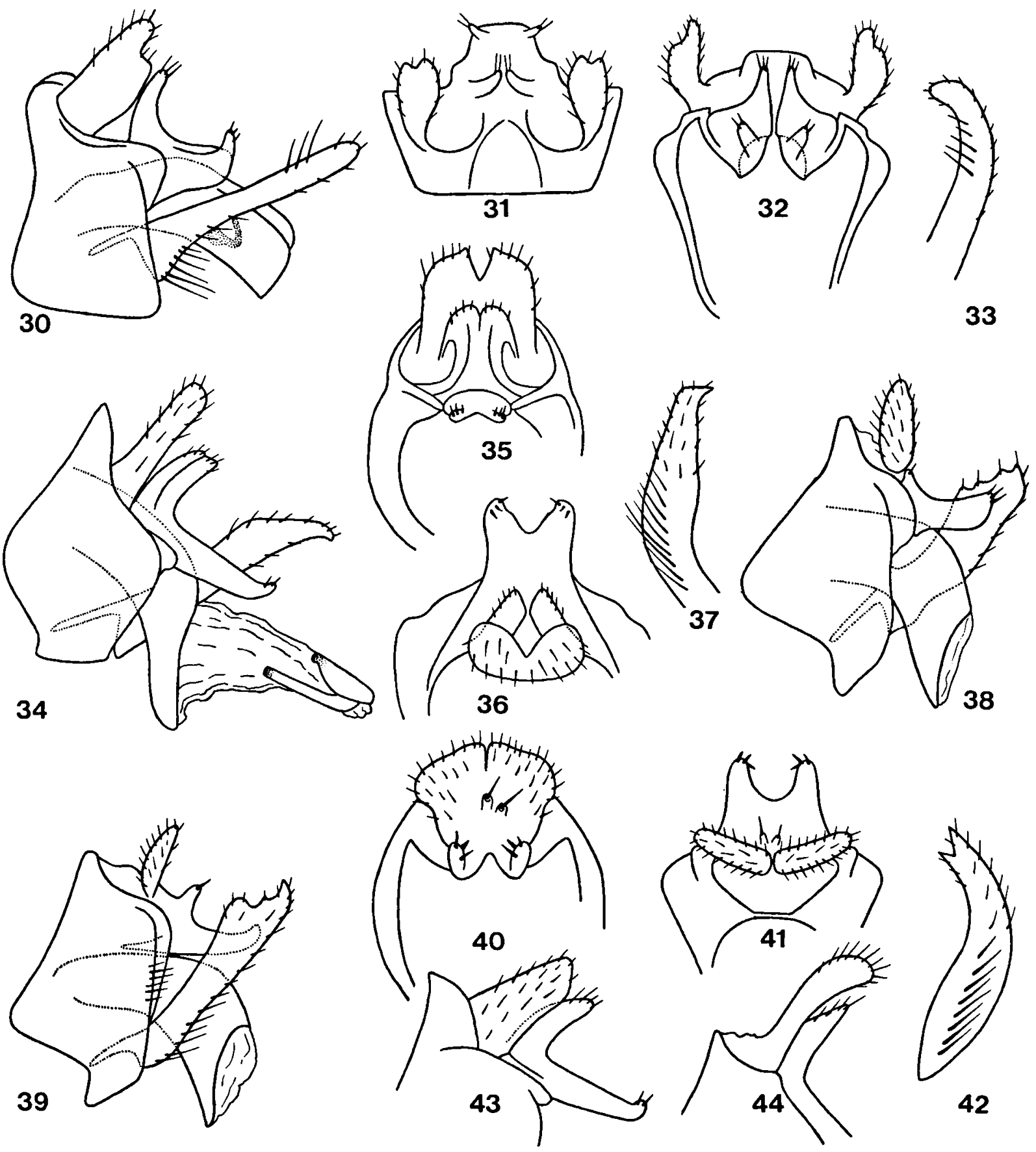

Figs. 30-33. Micrasema avadhiritam n. sp.: 30, génitalia de profil; 31, Xe segment de dessus; 32, Xe segment de face; 33, appendice inférieur de dessous.

Figs. 34-37. Micrasema jihmam n. sp.: 34, génitalia de profil; 35, Xe segment de face; 36, Xe segment de dessus; 37, appendice inférieur de dessous.

Fig. 38. Micrasema abhavyam n. sp.: génitalia de profil.

Figs. 39-42. Micrasema kripanam n. sp.: 39, génitalia de profil; 40, Xe segment de face; 41, Xe segment de dessus; 42, appendice inférieur de dessous.

Figs. 43-44. Micrasema jihmam n. sp.: variations du Xe segment de profil. 
Remarques. - M. avadhiritam (sanscrit, méprisé) est facile à reconnaître à son $\mathrm{Xe}$ segment en $\mathrm{V}$ et quadrilobé et à ses longs appendices inférieurs régulièrement grêles. Par la forme du Xe segment, elle s'apparente à $M$. jihmam n. sp. et à $M$. kripanam n. sp.

Cette espèce ne m'est connue que de quelques localités des Khasi Hills et de l'Etat de Manipour, où je l'ai capturée en décembre, mars, avril et mai.

Micrasema jihmam n. sp. (Figs. 34-37, 43-44)

Matériel. - Holotype $O^{\prime \prime}$ et allotype $Q$ : Sikkim, Teng; 12-V-1959 (CNC 21295). Paratypes dans CNC et ZMA.

Description. - Palpes maxillaires du mâle pas très longs, avec leur extrémité atteignant le niveau du $2 \mathrm{e}$ article des antennes.

Nervulation: chez les deux sexes, cellule discoïdale des ailes antérieures environ trois fois plus longue que large. Aux ailes postérieures, chez le mâle, le SR est tribranché et $M$ simple; chez la femelle, le $\mathrm{SR}$ est également tribranché et $\mathrm{M}$ double. VIe sternite du mâle sans pointe ventrale. Longueur de l'aile antérieure: $4,5-5,5 \mathrm{~mm}$.

Génitalia O (Figs. 34-37, 43-44): IXe segment assez allongé et avec ses deux angles latéraux, antérieur et postérieur, symétriquement arrondis et situés au même niveau; son bord latéral apical n'est pas replié vers l'intérieur et ne forme pas d'angle proéminent encadrant la phallocrypte. Appendices préanaux accolés l'un à l'autre sur les deux tiers de leur longueur, plus longs que larges, non échancrés et dressés verticalement. Xe segment assez grêle et de forme dérivée de celle d'un L; il se compose de deux branches basales, accolées l'une à l'autre, se montrant arrondies à leur extrémité lorsqu'elles sont vues de face et dressées verticalement sous les appendices préanaux auxquels elles sont parallèles. Il y a également une branche terminale en forte plaque horizontale, un peu oblique vers le bas et échancrée en triangle à son extrémité; les deux lobes ainsi formés sont arrondis à l'apex et pourvus d'épines dirigées vers le haut. Appendices inférieurs uni-articulés, grêles, amincis à leur extrémité qui est aiguë et recourbée vers l'intérieur et le bas; leur bord supérieur forme un bombement préapical bien caractéristique de l'espèce. Phallothèque tronquée très obliquement vers le bas à l'extrémité de sa face dorsale.

Remarques. - M. jihmam (sanscrit, louche) se reconnaît aisément à l'ensemble subvertical formé par les appendices préanaux et les branches basales du Xe segment accolées, par la longue branche apicale du même segment et par le bombement subapical supérieur des appendices inférieurs. Elle est voisine de $M$. kripanam $\mathrm{n}$. sp. et $M$. avadhiritam $\mathrm{n}$. sp. par la forme branchée du Xe segment.

Cette espèce est la seule du genre à avoir une large répartition géographique en Inde. Elle est commune au Sikkim, où je l'ai capturée de fin avril à mi-septembre. En Kameng, je l'ai rencontrée de fin mai à mi-juillet et dans l'Etat de Manipour de fin juin à mi-août. Je la connais aussi de quelques localités isolées des Khasi et Lushai Hills. J'ai noté une certaine variabilité dans la forme de l'ensemble dorsal quadrilobé (Figs. 43-44), de même que dans la profondeur de l'échancrure apicale de la branche terminale du Xe segment.

Micrasema kripanam n. sp. (Figs. 39-42)

Matériel. - Holotype $\sigma^{\circ}$ et allotype $\$$ : Inde, Assam, NEFA, Kameng Frontier Division, Dirang Dzong; 21/22-VII-1961 (CNC 21299). Paratypes dans CNC et ZMA.

Description. - Palpes maxillaires du mâle pas très longs, avec leur extrémité atteignant le niveau du $2 \mathrm{e}$ article des antennes.

Nervulation: aux ailes antérieures, cellule discoïdale, 1,5 fois plus longue que large chez le mâle et 2 fois chez la femelle. Aux ailes postérieures du mâle, SR est tribranché et $M$ simple. Chez la femelle, SR est tribranché et $M$ double. VIe sternite du mâle sans pointe ventrale. Longueur de l'aile antérieure: $4,5-5,5 \mathrm{~mm}$.

Génitalia $O^{\prime \prime}$ (Figs. 39-42): IXe segment plutôt court latéralement et avec son angle latéral apical arrondi et situé relativement haut et son angle latéral antérieur assez bas; son bord latéral apical n'est pas recourbé vers l'intérieur et ne forme pas d'angle proéminent encadrant la phallocrypte. Appendices 
préanaux petits, fusionnés l'un à l'autre sur toute leur longueur et dressés en plaque verticale; vus de face, ils apparaissent subrectangulaires. Xe segment apparaissant, en vue latérale, comme un ergot assez allongé et légèrement recourbé vers le haut à son extrémité; sa base forme un lobe très net et spineux; vu de dessus, le Xe segment se montre profondément échancré circulairement à son extrémité; les deux lobes ainsi formés sont arrondis, assez largement écartés l'un de l'autre et spineux à leur extrémité. Appendices inférieurs uni-articulés, grêles et s'épaississant graduellement jusqu'à leur extrémité qui est très obliquement tronquée et forme deux légères échancrures arrondies. Phallothèque avec une échancrure arrondie à son extrémité.

Remarques. - $M$. kripanam (sanscrit, misérable) présente le même Xe segment bifide que $M$. avadhiritam et $M$. jihmam, mais se distingue des autres espèces indiennes par ses appendices préanaux anguleux et fusionnés l'un à l'autre pour former une plaque verticale.

Je n'ai rencontré cette espèce qu'en Kameng, où elle ne paraît pas être rare, d'avril à septembre.

Micrasema abhavyam n. sp. (Fig. 38)

Matériel. - Holotype $\sigma^{\circ}$ : Sikkim, Chamiteng; 24-VIII-1959 (CNC 21304).

Description. - Cette espèce est très voisine de $M$. kripanam n. sp. et s'en distingue par la forme du Xe segment et des appendices inférieurs (Fig. 38). Celui-là montre son lobe baso-dorsal extrêmement réduit et son lobe apical fortement épaissi. Les ap- pendices inférieurs sont assez larges et avec leur bord inférieur nettement sinueux; l'apex de leur extrémité est tronqué obliquement vers le haut et légèrement dentelée. Longueur de l'aile antérieure $\mathrm{O}^{*}:$ 5,5 mm. Femelle inconnue.

Remarques. - $M$. abhavyam (sanscrit, malheureux) ne m'est connue que par le type, capturé au Sikkim oriental, vers $3000 \mathrm{~m}$ d'altitude. Je la considère comme distincte de $M$. kripanam à cause des deux caractères mentionnés plus haut et de sa provenance. Des recherches ultérieures montreront peut-être si ce n'est pas là une simple forme géographique de $M$. kripanam.

\section{Bibliographie}

Botosaneanu, L., 1976. Une collection de stades aquatiques de Trichoptères du Népal, réalisée par le Professeur H. Janetschek. Ergebn. Forsch.-Unternehmens Nepal Himalaya, 5: 187-200.

Martynov, A.V., 1909. Les Trichoptères du Thibet oriental et du Tsaidam, d'après les matériaux collectionnés par l'expédition de la Société impériale de Géographie de Russie, sous la direction de P.-K. Koslov. Annu. Mus. zool. Acad. imp. Sci. St. Pétersbourg (= Ezheg. zool. Mus.), 14: 256-309.

Mosely, M.E., 1938. The Indian Caddis Flies. J. Bombay nat. Hist. Soc., 40 (3): 486-496.

Schmid, F., 1961. Trichoptères du Pakistan IV. Tijdschr. Ent., 104(9): 187-230.

Schmid, F., 1963. Trichoptères d'Afghanistan. Annls. hist.-nat. Mus. natn. hung., 55 (Pars zool.): 435-445.

Schmid, F., 1989. Les Hydrobiosides (Trichoptera, Annulipalpia). Bull. Inst. r. Sci. nat. Belg. (Entomologie), 59 (Suppl.): 1-154, pls. I-XVIII.

Reçu: le 5 février 1992

Révision: le 28 avril 1992 